\title{
The Design of Internet of Things Environment Monitoring System Based
}

\section{on Data Fusion}

\author{
Qiang $\mathrm{Qi}^{1, \mathrm{a}}$, Bao-hua Jiang ${ }^{2, \mathrm{~b},{ }^{*}}$, Pei-xue $\mathrm{Liu}^{2, \mathrm{c}}$ and Fei Feng ${ }^{2, \mathrm{~d}}$ \\ ${ }^{1}$ Qingdao Binhai University Mechanical and Electrical Engineering College,China \\ ${ }^{2}$ Qingdao Huanghai University Mechanical and Electrical Engineering College,China \\ a66851766@qq.com, bjbh789@163.com, ${ }^{\text {'bytulpx@163.com, }{ }^{\mathrm{d}} 1547752800 @ q q . c o m}$
}

\begin{abstract}
This paper puts forward a kind of environmental monitoring system of internet of things based on data fusion technology. The system can real-time collect the temperature, humidity and other environmental parameters, wirelessly transmit the monitoring data through the wireless environmental monitoring network composed by ZigBee and GPRS technology, real-time monitors and processes the environmental parameters. In the study, the overall structure of the environmental monitoring system is introduced in detail, the hardware and software design of the system is analyzed, and the whole system is tested. The test results show that the system is stable and reliable, and the system has very important application value in the market.
\end{abstract}

Keywords: Data fusion, Environmental monitoring, ZigBee, GPRS.

\section{Introduction}

With the development of consumption level and the safety awareness of food and drug, on medical products, dairy products, seafood and other products, consumer not only pay attention to the variety and quantity, but also puts forward higher requirements on the quality of the products. China's transportation and warehousing environmental monitoring system uses only the early technology, monitoring technology in many storage and transportation are not fully used, compared with the international advanced level gap, because the service network and information system is not perfect, it greatly affect the accuracy and timeliness of warehousing, transportation and environment monitoring, there is a great potential safety problem the circulation of food, medicines and other products. At present, most of the storage and transportation enterprises in our country still use the traditional environmental monitoring system, to increase the independent environmental monitoring system in every enterprise, each warehouse or transport links, use a single monitoring software for each system, save the data to the user on a personal computer for GPRS mobile data acquisition also requires a static public IP address, there will be many drawbacks, such as: the authenticity of monitoring data is reliable? Every enterprise must have a set of software platform, the system cost is relatively high, increase the enterprise transportation and storage cost, effect the enthusiasm of the real monitoring of enterprises, and waste the repetitive formation of social resources. Therefore, the use of information technology, the establishment of environmental monitoring and early warning system of China's transportation warehousing system, real-time monitoring of food and other products in the process of transportation and environment, it is very important to ensure food safety ${ }^{[1]}$.

This paper presents an environmental monitoring system based on data fusion technology, it can real-time collect temperature, humidity and other environmental parameters, wirelessly transmit 
the monitoring data through wireless environmental monitoring network composed by ZigBee and GPRS technology, through the networking environment monitoring terminal equipment on their own, the user can conveniently monitor transportation and storage environment. The system is characterized by the optimization of the algorithm, small amount of computation, fast measurement speed, high data reliability, easy to operate.

\section{The design of environmental monitoring system}

1.1 The system structure

According to the information required to complete the task and multi network data fusion characteristics, the establishment of the system block diagram shown in figure 1.

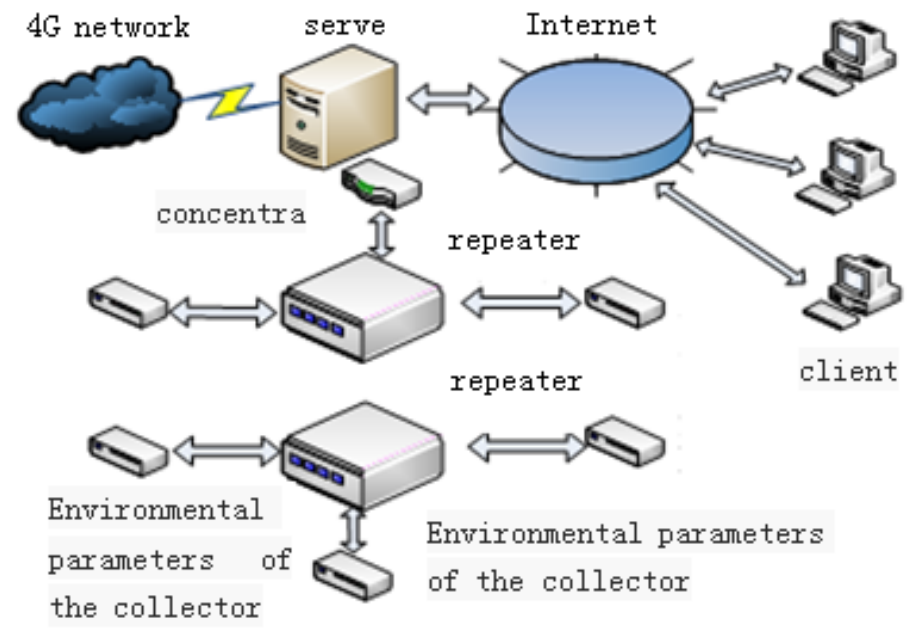

Fig.1 The overall frame

The system is mainly divided into two parts, monitoring terminal and cloud platform, terminal includes environment parameter collector and coordinator, the environmental parameters acquisition as the terminal nodes, includes repeater, through the ZigBee network information, the terminal nodes collect data to the coordinator, the coordinator reports to the cloud platform through the $4 \mathrm{G}$ network, the cloud platform receives the information uploading from the terminal and responses to client requirements ${ }^{[2]}$.

\subsection{System hardware design}

The hardware design of the environmental monitoring system includes the data acquisition part and the data transmission and control part of the anterior system. The overall block diagram of the system is shown in Figure 2. The hardware system is designed to two modules, so that it can fully meet the acquisition of the environmental temperature collection and data transmission and control.

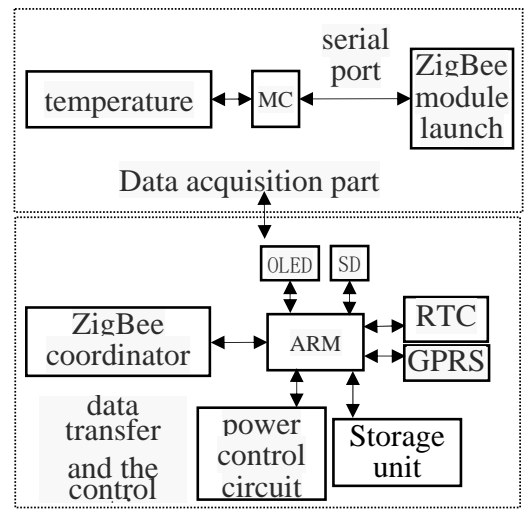

Fig.2 The system hardware diagram of the overall 
The data acquisition module is the front part of the whole system, which mainly realizes the function of data acquisition and wireless transmission of data. Temperature, humidity and dust data are collected through the sensor, and the data of each ZigBee collecting node in the environmental monitoring area is transmitted to the ZigBee coordinator through the control and processing of the single chip microcomputer. The communication between the ZigBee node and coordinator is through the wireless sensor network, and the ZigBee nodes cannot communicate with each other ${ }^{[3]}$.

The data transmission and control module is the central control part of the whole monitoring system. It mainly completes the receiving, storing and OLED data display of the previous data acquisition module. According to the overall requirements of the module, firstly receives data acquisition signal through the ZigBee coordinator, communicates with the ARM processor through a serial port, and transmits the data to the monitoring center by GPRS, then fulfils the whole wireless data transmission of the monitoring system.

\section{System software design}

The environmental monitoring system includes three parts, including data acquisition module design, data transmission and control module and host computer design.

\subsection{Software design of data acquisition module}

The data acquisition module is the front part of the environmental monitoring system, including the main program, data acquisition and processing of sensor network data transmission, data is sent to collect temperature, humidity and dust information package, the package is sent through wireless transmission by the node to the coordinator, connected by the network and the transmission and control module.

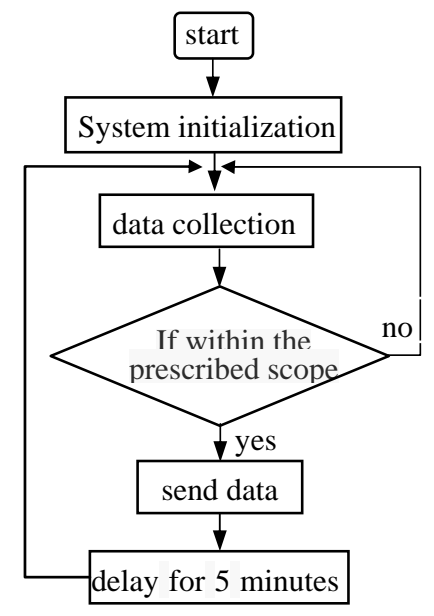

Fig.3 Data acquisition module flow chart

Temperature and humidity signal acquisition. The temperature and humidity sensors communication with MCU by Single bus mode, it works in low-power mode when no data transmission, when receiving the signal from the microcontroller send, converts the low power mode to high speed mode, after response, sensor data transmission bits, thereby completing a sensor.

The data transmission and control module is the core part of the whole control system. The software design of the system includes the transplantation of embedded operating system, the hardware driver and the application program.

Software design of monitoring center 
The software of the monitoring center mainly completes the function of data receiving, data processing and data management. The system management of the whole monitoring area can be achieved through the real-time data monitoring of the monitoring center. The monitoring center software is developed by Visual++6.0, which is used as a server in the data transmission process. Using winsock technology to achieve mutual communication, and through the database system to achieve the management of data.

The monitoring center software is divided into four parts, the display interface part, communication part, data fusion and data processing part. The interface includes the login interface and the monitoring interface, the communication part completes the communication initialization and connection, and data storage, data fusion part achieves the data fusion. The data processing part mainly realizes the function of data inquiry, chart analysis and report forms printing.

\section{Data fusion}

The data fusion refers to the data processing operation of computer simulation of the brain, according to the comprehensive treatment on a certain sequence of information from multiple sensors in certain criteria, so as to obtain accurate results to prevent misoperation.

\subsection{Fusion algorithm for recursive estimation based on mean}

For a limited time measurement, it can the estimate the measurement results through the value of available arithmetic mean, it is the results of unbiased estimation on measurement; because the detection cannot get all the data in real time, and thus cannot effectively use estimation algorithm. According to the characteristics of the system, a method of estimating the fusion algorithm based on the mean value of the partial recursive algorithm is proposed. The data fusion of batch estimation method is to divide the data into 2 groups, obtain the data fusion value of measured according to the theory of partial estimation ${ }^{[5]}$.

If the measurement $X$ data is divided into 2 groups: $\mathrm{x}_{11}, \mathrm{x}_{12}, \cdots, \mathrm{x}_{1 \mathrm{~m}}$ and $\mathrm{x}_{21}, \mathrm{x}$ $22, \cdots, \mathrm{x}_{2 \mathrm{k}}, \mathrm{m} \leqslant 4, \mathrm{k} \leqslant 4$, Calculates average $\mathrm{X}_{1} 、 \mathrm{X}_{2}$.

$$
\begin{aligned}
& \overline{X_{1}}=\frac{1}{m} \sum_{i=1}^{m} X_{1 i} \\
& \overline{X_{2}}=\frac{1}{K} \sum_{I=1}^{K} X_{2 i}
\end{aligned}
$$

The corresponding standard deviation is

$$
\begin{aligned}
& \sigma_{1}=\sqrt{\frac{1}{m-1} \sum_{i=1}^{m}\left(x_{1 i-} \overline{X_{1}}\right)^{2}} \\
& \sigma_{2}=\sqrt{\frac{1}{k-1} \sum_{i=1}^{k}\left(x_{2 i-} \overline{X_{2}}\right)^{2}}
\end{aligned}
$$

According to the theory of partial estimation, the variance of the fusion value is estimated as

$$
\begin{aligned}
& \sigma^{+}=\left[\left(\sigma^{-}\right)^{-1}+H^{T} R^{-1} H\right]^{-1} \\
& \sigma^{+2}=\sigma_{1}^{1} \sigma_{2}^{2} /\left(\sigma_{1}^{2} \sigma_{2}^{2}\right)
\end{aligned}
$$

The data fusion value of temperature and humidity 


$$
\begin{aligned}
& X^{+}=\frac{\sigma_{1}^{2} \sigma_{2}^{2}}{\sigma_{1}^{2}+\sigma_{2}^{2}}\left[\begin{array}{l}
1 \\
1
\end{array}\left[\begin{array}{cc}
1 / \sigma_{0}^{20} & \\
0 & 1 / \sigma_{0}^{2}
\end{array}\right]\left[\begin{array}{l}
-\frac{x_{1}}{x_{2}} \\
\end{array}\right]\right. \\
& =\frac{\sigma_{1}^{2} \sigma_{2}^{2}}{\sigma_{1}^{2}+\sigma_{2}^{2}}+\frac{\sigma_{1}^{2}}{\sigma_{1}^{2}+\sigma_{2}^{2}}
\end{aligned}
$$

Finally, bring the temperature and humidity values into the above formula, get the improved fusion value: temperature $(\mathrm{T}+)$ and humidity $(\mathrm{RH}+)$.

Fusion result analysis

In order to facilitate the customers to monitor the system, it can use PC develop client software or $\mathrm{B} / \mathrm{S}$ cloud server architecture by the use of PC monitoring system, it can view real-time data, history data query, report and a series of work. In order to reduce the amount of transmission data, the central node uses the mean to upload, at the server side, the measured data at some point are as follows:

Tab.1 Temperature and humidity measurements

\begin{tabular}{|c|c|c|c|c|}
\hline time & $\begin{array}{c}\text { center } \\
\text { node }\end{array}$ & $\begin{array}{c}\text { collector } \\
\text { number }\end{array}$ & $\begin{array}{c}\text { Temperature } \\
{\left[{ }^{\circ} \mathrm{C}\right]}\end{array}$ & $\begin{array}{c}\text { humidity } \\
{[\% \mathrm{RH}]}\end{array}$ \\
\hline 09:36:05 & 01 & 0101 & -14.9 & 85.6 \\
\hline 09:36:05 & 01 & 0102 & -15.0 & 85.5 \\
\hline 09:36:05 & 01 & 0103 & -14.9 & 85.5 \\
\hline 09:36:05 & 01 & 0104 & -14.8 & 85.5 \\
\hline 09:36:05 & 01 & 0105 & -15.0 & 85.4 \\
\hline 09:36:05 & 01 & 0106 & -14.9 & 85.5 \\
\hline 09:36:05 & 01 & 0107 & -14.9 & 85.6 \\
\hline 09:36:05 & 01 & 0108 & -14.9 & 85.6 \\
\hline 09:36:05 & 01 & 0109 & -14.9 & 85.6 \\
\hline 09:36:05 & 01 & 0110 & -14.9 & 85.5 \\
\hline
\end{tabular}

As can be seen from the table, after the data fusion the ZigBee terminal node take the mean, and the actual value of the error is small. At the same time found in the testing process, the transmission distance without relay without blocking is more than 100 meters, the through ability is stronger, through the monitoring system of data fusion, the less the amount of data transmission, due to the exclusion of invalid data, but also has higher accuracy.

\section{Summary}

This system will be applied to the data fusion technology of IOT in environmental monitoring, reduce the amount of data transmission, the data errors are eliminated, so that the data collected by the sensor can accurately and fast transmit to the server or data center, so the managers can easily get the site information, greatly improve the management efficiency, the system would be widely applied and promoted, video monitoring and remote control to the system will be the next research direction.

\section{Acknowledgement}

This research is supported by Shandong Provincial Science and Technology Project for Universities under Grant No.J16LN78. 


\section{References}

[1] Xiao Xinqing,Qi Lin,Zhang Lei.Wireless real time monitoring system for cold chain logistics of fresh grape[J],Application of electronic technology,2013,39(8):77-79.

[2]Wang Hongjun.Research on cold chain temperature monitoring system based on Wireless Sensor Networks [D]. ournal of Harbin University of Science and Technology,2011.

[3]Qi Lin,HanYubing,Zhang Xiaoshuang.Real time monitoring system of aquatic products cold chain logistics based on WSN [J],Chinese Journal of agricultural machinery,2012,43(8): 134-140.

[4]Li Zhi,Pei Junfang.A well distributed monitoring system based on Zigbee [J],bulletin of science and technology,2015,31(3):170-173.

[5]Yang Geng,Li Sen,Chen Zhengyu.High precision data fusion algorithm for privacy preserving in Sensor Networks[J],JournalofComputerScience,2013,36(1):189-195. 